\title{
FREE COMPLETELY REGULAR SEMIGROUPS
}

\author{
by P. G. TROTTER
}

(Received 5 May, 1983)

A completely regular semigroup is a semigroup that is a union of groups. The aim here is to provide an alternative characterization of the free completely regular semigroup $F_{X}^{c r}$ on a set $X$ to that given by J. A. Gerhard in $[3,4]$.

Although the structure theory for completely regular semigroups was initiated in 1941 [1] by A. H. Clifford it was not until 1968 that it was shown by D. B. McAlister [5] that $F_{X}^{c r}$ exists. More recently, in [7], M. Petrich demonstrated the existence of $F_{X}^{c r}$ by showing that completely regular semigroups form a variety of unary semigroups (that is, semigroups with the additional operation of inversion).

In [2] Clifford investigated the structure of $F_{X}^{c r}$ by considering it as a quotient semigroup $F_{X}^{u} / \rho$ of the free unary semigroup $F_{X}^{u}$ on $X$. He gave a simple description of Green's relations $\mathscr{L}, \mathscr{R}$ and $\mathscr{D}$ on $F_{X}^{u} / \rho$ and showed that $F_{X}^{\text {cr }}$ is a free semilattice of its $\mathscr{D}$-classes. The description of $\mathscr{D}$ was in terms of content of elements while the $\mathscr{L}$-class of an element was described modulo a description of the $\rho$-class of an element of lesser content. Clifford enabled the $\rho$-classes of elements of content at most 2 to be explicitly described, by providing a model for $F_{Z}^{c r}$ when $|Z|=2$.

J. A. Gerhard showed in [4] that an $\mathscr{H}$-class of $F_{X}^{u} / \rho$ is a free group. The free generators were described modulo solution of the word problem in $F_{X}^{u} / \rho$ for words of lesser content. With a given $\mathscr{H}$-class from each $\mathscr{D}$-class and with Petrich's description [6] of an arbitrary completely regular semigroup, Gerhard constructed a model for $F_{X}^{c r}$.

In this paper we inductively select a unique representative $w \theta \in F_{X}^{u}$ for each $\rho$-class $w \rho \in F_{X}^{u} / \rho$. In particular $w \theta$ is uniquely expressed as a product of elements from $X$ and from \{segments of $a \theta ; a \in F_{X}^{u}$ has smaller content than $w$ \}. It is then shown that the set $\left\{w \theta ; w \in F_{X}^{u}\right\}$ with the multiplication $u \theta \cdot v \theta=(u \theta(v \theta)) \theta$ is isomorphic to $F_{X}^{c r}$. Since they do not appear to shorten the proofs of this paper, the results of $[3,4]$ are not utilized here.

In section 1 some preliminary information, especially from [2], is listed. Some properties of $F_{X}^{u} / \rho$ are derived in section 2. In section $3, \theta$ is defined and relevant properties are derived. A model for $F_{\mathrm{X}}^{\mathrm{cr}}$ is obtained in the final section.

1. Definitions and preliminaries. Let $F_{X}$ and $F_{X}^{c r}$ denote respectively the free semigroup and free completely regular semigroup on a non-empty set $X$. Let $F_{X}^{u}$ denote the free unary semigroup on $X$; that is, the free object on $X$ in the category of semigroups with a unary operation. Let $\bar{X}=X \cup\left\{(,)^{-1}\right\}$ where $(,)^{-1} \notin X$. By [3], $F_{X}^{u}$ is the smallest subsemigroup of the free semigroup $F_{\bar{X}}$ such that $X \subseteq F_{X}^{u}$ and $(w)^{-1} \in F_{X}^{u}$ for all $w \in F_{X}^{u}$. There is an alternative description of $F_{X}^{u}$ in [2]. Let $F_{\bar{X}}^{1}$ and $F_{X}^{u 1}$ denote respectively the semigroups $F_{\bar{X}}$ and $F_{X}^{u}$ with identity 1 adjoined.

Define $v \in F_{\bar{X}}$ to be a segment of $w \in F_{X}^{u}$ if $w=a v b$ for some $a, b \in F_{X}^{1}$. The segment $v$

Glasgow Math. J. 25 (1984) 241-254. 
of $w$ is said to be maximal with respect to a property $P$ if and only if $v$ satisfies $P$ and for any final segment $c$ of $a$ and initial segment $d$ of $b$ then $c v, v d$ and $c v d$ do not satisfy $P$.

If $u=b(a \text { or } u=a)^{-1} b$ where $a \in F_{X}^{u 1}$ we say that the occurrence of (or $)^{-1}$ respectively is unmatched. Let $\mathbf{v} \in F_{X}^{u}$ denote the word obtained from $v \in F_{\bar{X}}$ by successively deleting unmatched occurrences of $(\text { and })^{-1}$.

The content of $w \in F_{\bar{X}}^{1}$ is the set

$$
C(w)=\{\text { letters of } X \text { appearing in } w\} .
$$

The left indicator $L(w)$ of $w \in F_{X}^{u}$ is given by $L(w)=\mathbf{a}$ where $a$ is the shortest initial segment of $w$ such that $C(a)=C(w)$. Dually the right indicator is $R(w)=\mathbf{b}$ where $b$ is the shortest final segment of $w$ such that $C(w)=C(b)$. Define $c$ to be an indicator of $w$ if there is a segment $c$ of $w$ such that $C(c)=C(w)$ and $L(\mathbf{c})=R(\mathbf{c})=\mathbf{c}$.

For $w \in F_{X}^{u}$ let $\left\{c_{j} ; 1 \leq j \leq r\right\}$ denote the set of successive segments of $w$ such that $c_{j}$ is an indicator and $c_{j}$ is not derivable from a proper subsegment of $c_{j}$. Let $d_{j}$ denote the final segment of $w$ beginning with $c_{j}$, and let $e_{j}$ denote the segment beginning and ending with $c_{j}$ and $c_{j+1}$ respectively. Define $I_{j}(w)=\mathbf{c}_{j}, W_{i}(w)=\mathbf{d}_{j}$ and $M_{j}(w)=\mathbf{e}_{j}$ to be respectively the $j$ th indicator, $j$ th remainder and $j$ th link of $w$.

EXAMPLE 1.1. Let $w=x_{1}\left(x_{2}\left(\left(x_{4} x_{1}\right)^{-1} x_{3}\right)^{-1}\left(x_{2}\right)^{-1} x_{1} x_{4} x_{3}\right)^{-1}$. Then

$$
\begin{aligned}
L(w) & =x_{1} x_{2}\left(x_{4} x_{1}\right)^{-1} x_{3}, \quad R(w)=x_{2} x_{1} x_{4} x_{3}, \quad I_{1}(w)=x_{2}\left(x_{4} x_{1}\right)^{-1} x_{3}, \\
I_{2}(w) & =x_{4} x_{1} x_{3} x_{2}, \quad I_{3}(w)=x_{3}\left(x_{2}\right)^{-1} x_{1} x_{4}, \quad I_{4}(w)=x_{2} x_{1} x_{4} x_{3}, \\
M_{1}(w) & =x_{2}\left(\left(x_{4} x_{1}\right)^{-1} x_{3}\right)^{-1} x_{2}, \quad M_{2}(w)=x_{4} x_{1} x_{3}\left(x_{2}\right)^{-1} x_{1} x_{4}, \quad M_{3}(w)=x_{3}\left(x_{2}\right)^{-1} x_{1} x_{4} x_{3}, \\
W_{1}(w) & =x_{2}\left(\left(x_{4} x_{1}\right)^{-1} x_{3}\right)^{-1}\left(x_{2}\right)^{-1} x_{1} x_{4} x_{3}, \ldots, W_{4}(w)=x_{2} x_{1} x_{4} x_{3} .
\end{aligned}
$$

Note that $I_{j}(w)=L\left(W_{j}(w)\right)=L\left(M_{j}(w)\right)=R\left(M_{j-1}(w)\right)$ (if the links exist) and $I_{1}(w)=$ $R(L(w))$.

We next provide a simple characterization of indicators and links. Suppose $v \in F_{X}^{u}$ and $C(v)=Y$. For any $u \in F_{X}^{u 1}$ and $x, y \in Y \backslash C(u)$ define $v$ to be

(i) a left [right] $Y$-indicator if $v=u y[v=x u]$,

(ii) a $Y$-indicator if $v=x u y, x \neq y$ (or if $v=x$ when $|Y|=1$ ), or

(iii) a Y-link if $v=x u x$.

LEMMA 1.2. Let $v \in F_{X}^{u}$ and $C(v)=Y$. Then $v$ is a left or right $Y$-indicator, $Y$-indicator or Y-link if and only if $v$ is a left or right indicator, indicator or link respectively of some $w \in F_{X}^{u}$.

Proof. Let $v=x u x$ be a $Y$-link as in the definition. Since $C(u) \neq Y$ and $x \in Y \backslash C(u)$ then $L(v)$ and $R(v)$ are successive indicators of $v$, so $v$ is a link of itself. Conversely let $M$ be the $j$ th link of $w$ where $C(w)=Y$. So $M$ has exactly two indicators, namely $I_{j}(w)=x a$ and $I_{j+1}(w)=b y$ for some $a, b \in F_{X}^{u 1}, x \in Y \backslash C(a)$ and $y \in Y \backslash C(b)$. So $M=x c y$ for some $c \in F_{X}^{u 1}$. If $C(c)=C(w)$ then $R(L(c))$ and $L(R(c))$ are indicators of $w$. Hence since $I_{j}(w)$ and $I_{j+1}(w)$ are successive indicators then $C(c) \neq C(w)$ while $C(x c)=C(w)=C(c y)$. But 
then, since $x \in C(c y)$, if $x \neq y$ we get $x \in C(c)$ and $C(c)=C(w)$, a contradiction. Thus $x=y$ and $M$ is a $Y$-link. The other cases follow directly from the definitions.

Let $\rho$ be the least congruence on $F_{X}^{u}$ containing $\left(w(w)^{-1} w, w\right),\left(w(w)^{-1},(w)^{-1} w\right)$ and $\left(\left((w)^{-1}\right)^{-1}, w\right)$ for all $w \in F_{X}^{u}$.

THEOREM 1.3. (Clifford [2]). Let $u, v \in F_{X}^{u}$. Then

(i) $F_{X}^{\mathrm{cr}} \cong F_{X}^{u} / \rho$,

(ii) $u \rho \mathscr{D} v \rho$ if and only if $C(u)=C(v)$, and

(iii) $u \rho \mathscr{R} v \rho$ if and only if $L(u)=a x$ and $L(v)=b x$ for some $x \in X$ and $a, b \in F_{X}^{u 1}$ such that $a \rho b$ or $a=b=1$.

CoROLlarY 1.4. Suppose $u, v \in F_{X}^{u}$ and $p$ is an initial segment of $u$. If $u \rho v$ then $v$ has an initial segment $q$ such that $L(\mathbf{p}) \rho L(\mathbf{q})$.

Proof. Assume $1 \neq|C(u)| \neq|C(p)|$ : otherwise the result follows directly from the theorem. Let $u, v, a, b$ be as in Theorem 1.3(iii). So $a \rho b$ and by [2, Lemma 5.1], $a \rho \mathbf{p} a_{1}$ for some $a_{1} \in F_{X}^{u 1}$. Since $|C(a)|<|C(u)|$ the result follows by induction on $|C(u)|$.

Notation. For $w \in F_{X}^{u}$ define $w^{n}$ to be the product of $n$ copies of $w$. Define $w^{-1}=(w)^{-1}, w^{-n}$ to be the product of $n$ copies of $w^{-1}$ and $w^{0}=w w^{-2} w$.

Throughout the paper assume that $X$ is a well ordered set. We will always denote by $Y$ the subset $Y=\left\{x_{1}, \ldots, x_{n}\right\}$ of $X$ where $x_{i}<x_{j}$ in $X$ if and only if $i<j$. Define

$$
\hat{f}=x_{1} \ldots x_{n} \text { and } f=\hat{f}^{0} \text {. }
$$

The symbol $\subset$ denotes proper inclusion of sets.

2. Some $\rho$-relationships. In this section we determine some relationships in $F_{X}^{u} / \rho$ and review Clifford's models for $|X| \leq 2$.

LEMMA 2.1. Let $w \in F_{X}^{u}$ and $u, v \in F_{X}^{u 1}$ such that $C(u v) \subseteq C(w)$. Let $a=$ $L(w) u(v R(w) w L(w) u)^{-1} v R(w)$. Then $w^{-1} \rho a$.

Proof. Clearly (aw) $\rho$ is an idempotent. By Theorem 1.3(iii) and its dual $a \rho \mathscr{H} w \rho \mathscr{H}(a w) \rho$. So awa $\rho a$ and waw $\rho$ w. By Theorem $1.3(\mathrm{i}), w^{-1} \rho$ is the unique $\mathscr{H}$-related inverse of $w \rho$ so $a \rho w^{-1}$.

Lemma 2.2. Let $w \in F_{X}^{u}$ have an initial segment $u$. Then $u^{-1} w \rho w$.

Proof. By [2, Lemma 5.1], $w \rho \mathbf{u} v$ for some $v \in F_{X}^{u 1}$.

The next lemma is the major step towards a decomposition of elements of $F_{X}^{u}$ in terms of their left and right indicators, indicators and links.

LEMma 2.3. Suppose $w \in F_{X}^{u}$ has no segment $u^{-1}$ such that $u \in F_{X}^{u}$ and $C(u)=C(w)$. Let $I_{j}=I_{j}(w)$ and $M_{h}=M_{h}(w), 1 \leq j \leq r, 1 \leq h<r$, be respectively the indicators and links of w. Then

$$
w \rho L(w) I_{1}^{-1} M_{1} \ldots I_{r-1}^{-1} M_{r-1} I_{r}^{-1} R(w) .
$$


Proof. Let $W_{j}=W_{j}(w)$ be the $j$ th remainder of $w$. By the definitions of section 1 , $L\left(W_{j}\right)=I_{j}$ and since $L(R(w))=I_{r}$ then $W_{r}=R(w)$. Furthermore since $W_{j}=W_{i}$ then there is a $z_{j} \in X$ and $w_{j} \in F_{X}^{u 1}$ such that $W_{i}=z_{j} w_{i}$. Then $I_{i+1}=R\left(L\left(w_{i}\right)\right)$ and $M_{i}=z_{j} L\left(w_{j}\right)$ (if they exist).

If $w=L(w) a$ for some $a$ then since $I_{1}=R(L(w))$ we get $W_{1}=I_{1} a$ and, by the dual of Lemma 2.2, $w \rho\left(L(w) I_{1}^{-1} I_{1}\right) a=L(w) I_{1}^{-1} W_{1}$. Alternatively $w=c d^{-1} e$ for some $c, d, e \in$ $F_{X}^{u 1}$ where $C(d) \neq C(w)$ and $L(w)=c \mathbf{g}$ for some inital segment $\mathrm{g}$ of $d^{-1}$. Since $I_{1}=$ $R(L(w))$ and $C(w) \neq C(d) \supseteq C(g)$ then $I_{1}=h \mathrm{~g}$ and $W_{1}=h d^{-1} e$ for some $h$. So by Lemma 2.2 and its dual

$$
\begin{aligned}
w \rho c\left(\mathrm{gg}^{-1} d^{-1} e\right) & =L(w) \mathbf{g}^{-1} d^{-1} e \rho\left(L(w) I_{1}^{-1} I_{1}\right) \mathbf{g}^{-1} d^{-1} e \\
& =L(w) I_{1}^{-1} h\left(\mathbf{g g}^{-1} d^{-1}\right) e \rho L(w) I_{1}^{-1} h d^{-1} e=L(w) I_{1}^{-1} W_{1}
\end{aligned}
$$

Hence we have $w \rho L(w) I_{1}^{-1} W_{1}$. If $r>1$ then by applying the argument to $w_{1}$ and using the initial comments of the proof, $w \rho L(w) I_{1}^{-1} M_{1} I_{2}^{-1} W_{2}$. Since $W_{r}=R(w)$ we get the result by repeating the argument for $w_{2}, \ldots, w_{r-1}$.

Recall the definition of $Y$ and $f$ in section 1 .

Corollary 2.4. Let $w$ be as in Lemma 2.3 with $C(w)=Y$. Then

$$
w \rho L(w) f\left(f I_{1} f\right)^{-1} f M_{1} f \ldots\left(f I_{r-1} f\right)^{-1} f M_{r-1} f\left(f I_{r} f\right)^{-1} f R(w) .
$$

Proof. Since $I_{1}=R(L(w))$ then by Theorem 1.3(iii) the idempotent $\left(f\left(f I_{1} f\right)^{-1} f I_{1}\right) \rho$ is $\mathscr{L}$-related to $(L(w)) \rho$. Hence since $I_{1}=L\left(M_{1}\right)$ then by Lemma 2.2

$$
L(w) I_{1}^{-1} M_{1} \rho L(w) f\left(f I_{1} f\right)^{-1} f I_{1} I_{1}^{-1} M_{1} \rho L(w) f\left(f I_{1} f\right)^{-1} f M_{1} .
$$

Likewise $I_{j}=R\left(M_{j-1}\right)=L\left(M_{\mathrm{j}}\right)$ if $j \neq r$ so

$$
M_{j-1} I_{i}^{-1} M_{j} \rho M_{j-1} f\left(f I_{j} f\right)^{-1} f M_{j}
$$

and since $I_{\mathrm{r}}=R\left(M_{r-1}\right)=L(R(w))$ then

$$
M_{r-1} I_{r}^{-1} R(w) \rho M_{r-1} f\left(f I_{r} f\right)^{-1} f R(w) .
$$

The result is now a consequence of Lemma 2.3.

Notice that if $w \in F_{X}^{u}$ and $C(w)=Y$ then by Lemma 2.1

$$
w^{-1} \rho L(w) f(f R(w) w L(w) f)^{-1} f R(w) .
$$

Hence by Corollary 2.4 any $w \in F_{X}^{u}$ where $C(w)=Y$ can be expressed modulo $\rho$ as a product of left and right $Y$-indicators, $Y$-indicators, $Y$-links and $\hat{f}$.

Lemma 2.5. Suppose $u, v, w \in F_{X}^{u 1}, C(w)=Y$ and $1<i \leq n, 1 \leq j<n$.

(i) If $w=u x_{1} \ldots x_{j}$ and $R(w)=v x_{1} \ldots x_{j}$ then $w \rho u f(f v f)^{-1} f R(w)$.

(ii) If $w=x_{i} \ldots x_{n} u x_{1} \ldots x_{i}$ then $w \rho x_{i} \ldots x_{n} u f(f u f)^{-1} f u x_{1} \ldots x_{i}$.

Proof. (i) By Theorem 1.3 the idempotent $\left(x_{i+1} \ldots x_{n} \hat{f}^{-1}(f v f)^{-1} f R(w)\right) \rho$ is $\mathscr{L}$-related 
to $w \rho$. So

$$
w \rho w x_{j+1} \ldots x_{n} \hat{f}^{-1}(f v f)^{-1} f R(w) \rho u f(f v f)^{-1} f R(w) .
$$

(ii) Let $R(w)=v x_{1} \ldots x_{j}$. Since $R\left(f u x_{1} \ldots x_{i}\right)=R(w)$ then by $w \rho x_{i} \ldots x_{n} u f(f v f)^{-1} f R(w)$ and $f u x_{1} \ldots x_{j} \rho f u f(f v f)^{-1} f R(w)$. The result follows.

Definition 2.6. (i) A segment $u$ of $w \in F_{X}^{u}$ is $v$-excluded, for $v \in F_{\bar{X}}$, if and only if $v$ is not a subsegment of $u$.

(ii) An $\hat{f}$-excluded segment $u$ of $w \in F_{X}^{u}$ is $\hat{f}$-bounded if and only if either $w=u$, $w=a \hat{f} u, w=u \hat{f} b$ or $w=a \hat{f} u \hat{f} b$ for some $a, b \in F_{X}^{u 1}$.

(iii) Denote by $G_{Y}$ the free group freely generated by

$$
\left\{f u f, \hat{f} ; u \in F_{X}^{u}, C(u) \subseteq Y \text { and } u \text { is } \hat{f} \text {-excluded }\right\}
$$

where $(f u f)^{-1}$ and $\hat{f}^{-1}$ denote respectively the inverses of $f u f$ and $\hat{f}$, and $f$ is the identity. If $Y=\{x\}$ then $\hat{f}=x$, so there exists no $\hat{f}$-excluded $u \in F_{X}^{u}$ such that $C(u) \subseteq Y$. Hence $G_{\{x\}}$ is the free group on $\{x\}$. Let $G_{f}$ denote the subgroup of $G_{Y}$ generated by $\hat{f}$. We will regard $v \in F_{X}^{u}$ as an alternative expression for $u \in G_{Y}$ if and only if a common expression can be obtained by replacing segments of $u$ and $v$ that are words of $G_{f}$ by their reduced forms. For example $(f a \hat{f})^{-1} f b \hat{f}^{-1}$ denotes $(f a f \hat{f})^{-1} f b f \hat{f}^{-1}=\hat{f}^{-1}(f a f)^{-1} f b f \hat{f}^{-1}$ in $G_{Y}$.

EXAMPLE 2.7. (i) Let $X=\{x\}$. By [2] $F_{X}^{c r} \cong G_{X}$. Let $w \theta$ be the reduced form in $G_{X}$ of $w \in F_{X}^{u}$. By [2], for any $u, v \in F_{X}^{u}, u \rho v$ if and only if $u \theta=v \theta$. So $G_{X}=\left\{w \theta, w \in F_{X}^{u}\right\}$ with multiplication given by $u \theta \cdot v \theta=(u \theta(v \theta)) \theta$.

(ii) Let $X=Y=\{x, y\}$, so $\hat{f}=x y$. Let $A=\left\{x^{j}, y^{j} ; j\right.$ is a non-zero integer $\}$ and $H_{Y}$ be the subgroup of $G_{Y}$ freely generated by $\{f u f, \hat{f} ; u \in A\}$. Let $D_{x}=G_{\{x\}}, D_{y}=G_{\{y\}}$ and

$$
D_{x y}=\left\{p f h f q ; p \in A \cup\left\{y^{0}, 1\right\}, q \in A \cup\left\{x^{0}, 1\right\}, h \in H_{Y}\right\} .
$$

Let $S=D_{x} \cup D_{y} \cup D_{x y}$. Note that $x^{0} f \rho f \rho f y^{0}$ and for integers $i, j$ that $x^{i} y^{i} \rho x^{i-1} f f f y^{j-1}$ and by Lemma 2.5 (ii) $y^{i} x^{j} \rho y^{i} f f x^{j}$. With these relations we can uniquely choose $w \theta \in S$ such that $w \rho w \theta$ for all $w \in F_{X}^{u}$. It follows easily from [2, section 6] that for $u, v \in F_{X}^{u}, u \rho v$ if and only if $u \theta=v \theta$ and $F_{X}^{\mathrm{cr}} \cong S=\left\{u \theta ; u \in F_{X}^{u}\right\}$ with multiplication $u \theta \cdot v \theta=(u \theta(v \theta)) \theta$. The $\mathscr{D}$-classes of $S$ are $D_{x}, D_{y}$ and $D_{x y}$ and $H_{Y}$ is the $\mathscr{H}$-class of $f$.

3. $\theta$-forms. An element $w \theta \in F_{X}^{u}$ will be constructed from any $w \in F_{X}^{u}$. It will be shown that $w \theta \rho w, w \theta \theta=w \theta$ and for $u, v \in F_{X}^{u}$ that $(u v) \theta=(u \theta(v \theta)) \theta$. These properties will be used in the next section to show that $S=\left\{w \theta ; w \in F_{X}^{u}\right\}$, under the multiplication $u \theta \cdot v \theta=(u \theta(v \theta)) \theta$, is a semigroup isomorphic to $F_{X}^{c r}$, and that $w \theta$ is a unique representative of the $\rho$-class $w \rho$.

The construction of $w \theta$ will depend on the following assumption. Recall the definition of $Y$ and $f$ from section 1 .

Assumption 3.1. In the remainder of the paper assume for each $w \in F_{X}^{u}$ where $C(w) \subset Y$ that a unique representative $w \theta$ of the $\rho$-class $w \rho$ has been constructed. In particular if $|C(w)| \leq 2$ let $w \theta$ be as in Examples 2.7 . 
The following definition is needed for our selection of representatives of the $\rho$-classes $f w$, $w f$ and $f w f$, where $C(w) \subset Y$.

Defintrion 3.2. Define $x_{0}=x_{n+1}=1$. For $w \in F_{X}^{u}$ where $C(w) \subset Y$ let $i(w)$ and $j(w)$ be respectively the least and greatest integer such that whenever $0 \leq i \leq j(w)$ or $i(w) \leq i \leq$ $n+1$ then $x_{i} \in C(w) \cup\{1\}$. Define $w_{L} \in F_{X}^{u 1}$ to be the shortest initial segment of $\left(w\left(x_{0} \ldots x_{j(w)}\right)^{0}\right) \theta$ such that $w x_{0} \ldots x_{j(w)} \rho w_{L} x_{0} \ldots x_{j(w)}$. Define $w_{R} \in F_{X}^{u 1}$ dually to be the shortest final segment of $\left(\left(x_{i(w)} \ldots x_{n+1}\right)^{0} w\right) \theta$ such that $x_{i(w)} \ldots x_{n+1} w \rho x_{i(w)} \ldots x_{n+1} w_{R}$. Define

$$
w_{l}=\left[\left(x_{i(w)} \ldots x_{n+1}\right)^{0} w\right]_{L}, \quad w_{r}=\left[w\left(x_{0} \ldots x_{j(w)}\right)^{0}\right]_{R} \quad \text { and } \quad w_{M}=w_{l r} .
$$

The next lemma indicates the need for Definition 3.2. To facilitate its proof we make another assumption.

Assumption 3.3. Suppose $v \in F_{X}^{u}, u=\left(x_{i}, \ldots x_{n+1}\right)^{0} v$ for some $i$ and $Y \supset C(u) \supset C(v)$. Assume that $v_{R}$ is a final segment of $u \theta$. Dually assume that if $u=v\left(x_{0} \ldots x_{i}\right)^{0}$ for some $j$ then $v_{\mathrm{L}}$ is an initial segment of $u \theta$.

The assumption can be seen to be valid, by Examples 2.7, if $|C(w)| \leq 2$. We will define $\theta$ such that the assumption will be valid when $C(u)=Y$ (see comment after Lemma 3.11).

Lemma 3.4. Suppose $v, w \in F_{X}^{u 1}$ and $C\left(x_{i} \ldots x_{n+1} w x_{0} \ldots x_{j}\right) \subset Y$ for some $i, j$. Then

(i) $w f \rho w_{L} f, f w \rho f w_{R}$ and $f w f \rho f w_{M} f$;

(ii) if $w x_{0} \ldots x_{j} \rho v x_{0} \ldots x_{j}$ then $w_{L}=v_{L}$;

(iii) if $x_{i} \ldots x_{n+1} w x_{0} \ldots x_{i} \rho x_{i} \ldots x_{n+1} v x_{0} \ldots x_{j}$ then $w_{M}=v_{M}$;

(iv) $w_{L L}=w_{L}, w_{L M}=w_{R M}=w_{M M}=w_{M}$.

Proof. (i) Since $\left(x_{0} \ldots x_{j(w)}\right)^{0} f \rho f$ and $w\left(x_{0} \ldots x_{j(w)}\right)^{0} \rho w_{L}\left(x_{0} \ldots x_{j(w)}\right)^{0}$ then $w f \rho w_{L} f$ and dually $f w \rho f w_{R}$. Hence (with duals) $f w_{L} f \rho f\left(x_{i(w)} \ldots x_{n+1}\right)^{0} w f \rho f w f$, so $f w_{M} f=$ $f w_{l r} f \rho f w_{t} f \rho f w f$.

(ii) Since $C\left(x_{i} \ldots x_{n+1} w x_{0} \ldots x_{i}\right) \subset Y$ then $0 \leq j<i \leq n+1, i \neq 1$ and $j \neq n$. Suppose $v x_{0} \ldots x_{j} \rho w x_{0} \ldots x_{j}$. Assume $j(w) \geq j(v)$. Also assume $j \geq j(w)$; otherwise multiply both sides of the relation by suitable elements of $Y$. Since $x_{j(w)+1} \notin C(w)$ then $w x_{0} \ldots x_{j(w)+1}=$ $L\left(w x_{0} \ldots x_{j(w)+1}\right)$. If $j>j(w)$ then by Corollary 1.4 there is a segment $a$ of $v x_{0} \ldots x_{j}$ such that $L(\mathbf{a})=\mathbf{a} \rho w x_{0} \ldots x_{j(w)+1}$. Since $x_{j(v)+1} \notin C(v)$ it follows by Theorem 1.3(iii) that $\mathbf{a}=v x_{0} \ldots x_{j(w)+1}$ and $w x_{0} \ldots x_{j(w)} \rho v x_{0} \ldots x_{j(w)}$. So assume $j=j(w)$. The result is then immediate if $j(w)=j(v)$, so assume $j(w)>j(v)$. Then $C(v) \subset C(w)$ and by Assumption 3.3, $v_{L}$ is an initial segment of $\left(w\left(x_{0} \ldots x_{j}\right)^{0}\right) \theta$. But $v_{L} x_{0} \ldots x_{j} \rho v x_{0} \ldots x_{j} \rho w x_{0} \ldots x_{i}$, so $w_{L}$ is an initial segment of $v_{L}$. Since $C(v) \supseteq C\left(v_{L}\right)$ then $j\left(w_{L}\right) \leq j\left(v_{L}\right) \leq j(v)$. Since $v x_{0} \ldots x_{i} \rho w_{L} x_{0} \ldots x_{j}$ then as above $v_{L}$ and hence $w_{L}$ are initial segments of $w_{L L}$. By the definition of $w_{L L}$ this is possible only if $w_{L}=w_{L L}$. So $v_{L}=w_{L}$.

(iii) Let $\mathbf{U}=\left\{u ; x_{i} \ldots x_{n+1} u x_{0} \ldots x_{j} \rho x_{i} \ldots x_{n+1} w x_{0} \ldots x_{j}\right\}$. Select $a, b \in \mathbf{U}$ such that $i(a) \geq i(u)$ and $j(b) \leq j(u)$ for all $u \in \mathbf{U}$. We first prove the existence of $d \in \mathbf{U}$ such that $i(d)=i(a), j(d)=j(b)$. Suppose $j(a)>j(b)$; otherwise put $d=a$. So $x_{i(b)+1} \in C(a)$ and $a$ 
has a shortest initial segment $p$ that includes $x_{j(b)+1}$. So $\mathbf{p}=c x_{j(b)+1}=L(\mathbf{p})$ for some $c \in F_{X}^{u 1}$. By Corollary 1.4 and Theorem 1.3(iii) applied to $x_{i} \ldots x_{n+1} a x_{0} \ldots x_{j}$ and $x_{i} \ldots x_{n+1} b x_{0} \ldots x_{j}$ we get $x_{i} \ldots x_{n+1} c \rho x_{i} \ldots x_{n+1} b x_{0} \ldots x_{i(b)}$. It follows that with $d=$ $c\left(x_{0} \ldots x_{j(b)}\right)^{-1}$ then $d \in \mathbf{U}$. We have $i(c) \geq i(a) \geq i>j \geq j(b) \geq j(c)$ by the choice of $i(a)$ and $j(b)$, hence since $d \in \mathbf{U}$ then $i(d)=i(a)$ and $j(d)=j(b)$.

It sufficies to prove $w_{M}=d_{M}$; by the same proof $v_{M}=d_{M}$. As in the proof of (ii) we assume $j=j(w)$ and likewise $i=i(w)$. Since $\left(x_{i} \ldots x_{n+1}\right)^{0} w x_{0} \ldots x_{i} \rho\left(x_{i} \ldots x_{n+1}\right)^{0} d x_{0} \ldots x_{j}$ then by (ii), $w_{l}=\left(\left(x_{i} \ldots x_{n+1}\right)^{0} d\right)_{L}$. So $j(d)=j\left(\left(x_{i} \ldots x_{n+1}\right)^{0} d\right) \geq j\left(w_{l}\right)$. Since $w_{l} \in \mathbf{U}$ then by the choice of $d, j(d)=j\left(w_{l}\right)$. By these observations

$$
\begin{aligned}
x_{i} \ldots x_{n+1} w_{l}\left(x_{0} \ldots x_{j(d)}\right)^{0} & \\
= & x_{i} \ldots x_{n+1}\left(\left(x_{i} \ldots x_{n+1}\right)^{0} d\right)_{L}\left(x_{0} \ldots x_{j(d)}\right)^{0} \rho x_{i} \ldots x_{n+1}\left(x_{i} \ldots x_{n+1}\right)^{0} d\left(x_{0} \ldots x_{i(d)}\right)^{0} \\
& \rho x_{i} \ldots x_{n+1}\left(x_{i(d)} \ldots x_{n+1}\right)^{0} d\left(x_{0} \ldots x_{j(d)}\right)^{0} \rho x_{i} \ldots x_{n+1} d_{l}\left(x_{0} \ldots x_{i(d)}\right)^{0} .
\end{aligned}
$$

Since $w_{M}=\left(w_{l}\left(x_{0} \ldots x_{j(d)}\right)^{0}\right)_{R}$ and similarly for $d_{M}$, then by the dual of (ii), $w_{M}=d_{M}$.

(iv) We have $w_{L} x_{0} \ldots x_{j(w)} \rho w x_{0} \ldots x_{j(w)}$ and for $z=w_{L}, \quad w_{R}$ or $w_{M}$ then $x_{i(w)} \ldots x_{n+1} z x_{0} \ldots x_{j(w)} \rho x_{i(w)} \ldots x_{n+1} w x_{0} \ldots x_{i(w)}$. The result follows by (ii) and (iii).

We now extend Definition 3.2 to include some cases where $C(w)=Y$.

Defintrion 3.5. Let $u$ be a segment of $w \in F_{X}^{u}$ where $C(u)=C(w)=Y$. Let $v \in F_{X}^{u 1}$ and $x, y \in Y \backslash C(v)$. Define

$$
\begin{aligned}
& u_{\mathrm{L}}=v \theta y \quad \text { if } u=v y \text { is a left } Y \text {-indicator, } \\
& u_{R}=x(v \theta) \quad \text { if } u=x v \text { is a right } Y \text {-indicator, and } \\
& u_{M}= \begin{cases}x(v \theta) y & \text { if } u=x v y \text { is a } Y \text {-indicator or } Y \text {-link, } \\
v_{R} y & \text { if } u=v y \text { is a left (not right) } Y \text {-indicator, } \\
x v_{L} & \text { if } u=x v \text { is a right (not left) } Y \text {-indicator. }\end{cases}
\end{aligned}
$$

By Lemma 3.4 and Assumption 3.1 we easily get the following.

LEMMA 3.6. If they exist $u_{L L}=u_{L}, u_{R R}=u_{R}$ and $u_{M M M}=u_{M M}$ for any $u \in F_{X}^{u}$, $C(u) \subseteq Y$.

Suppose $w \in F_{X}^{u}$ and $C(w)=Y$. The following operations will be used in the selection of $w \theta$. Recall the definitions of section 1 and Definitions 2.6, 3.2 and 3.5.

$(\theta 1)$ Construct $w \theta_{1}$ from $w$ by replacing each segment that is maximal with respect to being a word in $G_{\{x\}}$, for any $x \in X$, by its reduced form in $G_{\{x\}}$. Clearly $w \theta_{1} \rho w$.

$(\theta 2)$ Construct $w \theta_{2}$ from $w \theta_{1}$ by replacing each segment $u^{-1}$, where $C(u)=Y$ and $\hat{f}^{i} \neq u \neq$ faf for any $i$ and $a$, by $L(u) f(f R(u) u L(u) f)^{-1} f R(u)$. Likewise replace segments $v^{-1}$ of $u$ where $C(v)=Y$, and so on. If $u=\hat{f}^{i}$ or faf and $u^{-1}$ is not preceded and succeeded by $\hat{f}$ in the spelling of $w$ then replace $u^{-1}$ by $f u^{-1} f$.

By (2), $w \theta_{2} \rho w$. Note that if $u^{-1}$ is a segment of $w \theta_{2}$ and $C(u)=Y$ then $u^{-1} \in G_{Y}$ and $\hat{f} u^{-1} \hat{f}$ is also a segment of $w \theta_{2}$.

( $\theta 3$ ) Construct $w \theta_{3}$ from $w \theta_{2}$ by replacing each $\hat{f}$-bounded segment $u$, where 
$C(u)=Y$ and $u$ has $r Y$-indicators, by

$$
L(u) f\left(f I_{1}(u) f\right)^{-1} f M_{1}(u) f \ldots\left(f I_{r-1}(u) f\right)^{-1} f M_{r-1}(u) f\left(f I_{r}(u) f\right)^{-1} f R(u) .
$$

By Corollary 2.4, $w \theta_{3} \rho w$. Note that $w \theta_{3}$ is a product in $F_{X}^{u}$ of $\hat{f}$ and $\hat{f}$-bounded segments. We see by Definitions 3.2 and 3.5 that $u_{M}$ exists for any $\hat{f}$-bounded segment $u$ of $w \theta_{3}$, where $u$ is not an initial or final segment; otherwise $u_{L}$ or $u_{R}$ respectively exist. This property is invariant under the operations on $w \theta_{3}$ that follow.

$(\theta 4)$ Construct $w \theta_{4}$ from $w \theta_{3}$ by replacing each $\hat{f}$-bounded segment $u=x_{i} a$, where $C(u)=Y, a$ has initial segment $p$ such that $\mathbf{p} \rho x_{i+1} \ldots x_{n+1}$ and $L(u)=x_{i} b \neq u$ for some $i$, by $L(u) f\left(f \mathbf{p}^{-1} b f\right)^{-1} f \mathbf{p}^{-1} a$. Since $u \rho x_{i} \ldots x_{n} \mathbf{p}^{-1} a$ then by the dual of Lemma $2.5(i)$, $w \theta_{4} \rho w$.

$(\theta 5)$ Construct $w \theta_{5}$ from $w \theta_{4}$ by replacing each $\hat{f}$-bounded segment $u=a x_{i}$, where $C(u)=Y$, a has final segment $q$ such that $\mathbf{q} \rho x_{0} \ldots x_{j-1}$ and $R(u)=b x_{j} \neq u$ for some $j$, by $a \mathbf{q}^{-1} f\left(f b \mathbf{q}^{-1} f\right)^{-1} f R(u)$. Then $w \theta_{5} \rho w$.

( $\theta 6)$ Construct $w \theta_{6}$ from $w \theta_{5}$ by replacing each $\hat{f}$-bounded segment $u=x_{i} a x_{j}$, where $a$ has initial and final segments $p$ and $q$ respectively such that $\mathbf{p} \rho x_{i+1} \ldots x_{n+1}$ and $\mathbf{q} \rho x_{0} \ldots x_{j-1}$ for some $i$ and $j$, by $x_{i} a \mathbf{q}^{-1} f\left(f \mathbf{p}^{-1} a \mathbf{q}^{-1} f\right) f \mathbf{p}^{-1} a x_{j}$. By Lemma 2.5(ii), $w \theta_{6} \rho w$.

( $\theta 7)$ Construct $w \theta_{7}$ from $w \theta_{6}$ by replacing each $\hat{f}$-bounded segment $u$ by $u_{L}, u_{R}$ or $u_{M M}$ according as $u$ is an initial, final or other type of segment. By Lemma 3.4(i) and Definition 3.5, $w \theta_{7} \rho w$.

( $\phi$ ) For $w \in F_{X}^{u}$ where $C(w)=Y$, construct $w \phi$ from $w$ by replacing the segment of $w$ that is maximal with respect to being a word of $G_{Y}$ by its reduced form in $G_{Y}$.

$(\theta)$ Define $w \theta=w \theta_{7} \phi$. Then $w \theta \rho w$.

Notice that each $\hat{f}$-bounded segment of $w \theta$ of content $Y$ is a left $Y$-, right $Y$ - or $Y$-indicator or $Y$-link. Furthermore $w \theta$ is a product in $F_{X}^{u}$ of $\hat{f}$ and $\hat{f}$-bounded segments. By $(\theta 3), w \theta$ has a segment that is a word in $G_{Y}$. We have $w \theta=p h q$ where $h \in G_{Y}, p=1$ or $p=u f$ and $q=1$ or $q=f v$ where $u$ and $v$ are respectively the $\hat{f}$-bounded initial and final segments (if they exist) of $w \theta$.

The next result follows easily from the definitions.

LEMma 3.7. Let $w=$ phkq where $h, k \in G_{Y}, p, q \in F_{X}^{u 1}$ and $C(w)=Y$. Then $w \theta=$ $((p h) \theta(k q) \theta) \phi$.

LEMMA 3.8. If $w \in F_{X}^{u}$ then $w \theta \theta=w \theta$.

Proof. The result is immediate by Assumption 3.1 if $C(w) \subset Y$ and it is easy to check that $h \theta=h$ for any $h \in G_{f}$ (see Definition 2.6(iii)) and that $u^{-1} \theta=(u \theta)^{-1}$ for any $u \in G_{\mathrm{Y}}$. Clearly $w \theta \theta_{2}=w \theta$. Assume $C(w)=Y$ and $v$ is an $\hat{f}$-bounded segment of $w \theta$. By $(\theta 7)$, $v=u_{L}, u_{R}$ or $u_{M M}$ for some $u \in F_{X}^{u}$ and by Lemma $3.6 v_{L}=v, v_{R}=v$ or $v_{M}=v$ respectively. Assume $\boldsymbol{w} \theta=v f$ or $f v f$ : by duality and Lemma 3.7 we need only prove the result in these cases. If $C(v) \subset Y$ then we easily see $w \theta \theta=w \theta$. So assume $C(v)=Y$. Since $(\theta 4),(\theta 5)$ and $(\theta 6)$ are used in the construction of $v$ it can be easily checked that if $v=x_{i} p a$ for some $i$, where $\mathbf{p} \rho x_{i+1} \ldots x_{n+1}$ then $L(v)=v$. Hence if $v$ is a segment of $w \theta \theta_{3}$ then $v$ is not modified by $(\theta 4)$; similarly $v$ is invariant under $(\theta 5)$ and $(\theta 6)$. If $w \theta=v f$ then 
$v=L(v)$, so $w \theta \theta_{3}=v f(f R(v) f)^{-1} f R(v) f$ and since $v$ is invariant under $(\theta 4),(\theta 5),(\theta 6)$ and $(\theta 7)$ then $w \theta \theta=w \theta$. Now suppose $w=f v f$. If $v$ is a left $Y$-, right $Y$-, or $Y$-indicator then we likewise get $w \theta \theta=w \theta$. Alternatively if $v$ is a $Y$-link then $w \theta \theta_{3}=$ $f L(v) f(f L(v) f)^{-1} f v f(f R(v) f)^{-1} f R(v) f$ and as above we get the result.

Reasoning in a similar way we get the following.

Corollary 3.9. If $w \in F_{X}^{u}$ and $C(w)=Y$ then $w \theta_{i} \theta=w \theta$ for $1 \leq i \leq 7$.

The last three results will be used several times without comment in the following lemmas. The next result is like Corollary 2.4 but without restrictions on inverses.

Lemma 3.10. Let $w \in F_{X}^{u} \quad$ where $C(w)=Y$ and $w=w \theta_{2}$. Then $w \theta=$ $\left(L(w) f\left(f I_{1}(w) f\right)^{-1} f W_{1}(w)\right) \theta$ and $(f w) \theta=\left(f L(w) f\left(f I_{1}(w) f\right)^{-1} f W_{1}(w)\right) \theta$. Furthermore if $W_{1}(w)=w \neq R(w)$ and $\hat{f}$ is not an initial segment of $w$ then $\left(f W_{1}(w)\right) \theta=$ $\left(f M_{1}(w) f\left(f I_{2}(w) f\right)^{-1} f W_{2}(w)\right) \theta$.

Proof. Let $b$ be the $\hat{f}$-bounded initial segment of $w$; if no such segment exists then, by $(\theta 2), w$ has initial segment $\hat{f}$ and the results follow. If $L(b)=L(w)$ then the expressions for $w \theta$ and $(f w) \theta$ are consequences of $(\theta 3)$. Suppose $L(b) \neq L(w)$. So $w=b \hat{f} c$ for some $b, c \in F_{X}^{u}$ such that $C(b) \subset Y$. Then $L(w)=b q x_{i}$ for some $j<n, q=x_{0} \ldots x_{j-1}$ and $R(L(w))=I_{1}(w)=d q x_{j}$ for some $d$. By $(\theta 5)$ and $(\phi), \quad(L(w) f) \theta=$ $\left(b q q^{-1} f\left(f d q q^{-1} f\right)^{-1} f d q x_{j} f\right) \theta$. Since $\left(b q q^{-1}\right)_{L}=b_{L}$ and $\left(d q q^{-1}\right)_{M}=d_{M}$ by Lemma 3.4(ii), (iii) then by $(\theta 7)(L(w) f) \theta=\left(b f(f d f)^{-1} f I_{1}(w) f\right) \theta$. We have $\left(f W_{1}(w)\right) \theta=(f d \hat{f} c) \theta=(f d f \hat{f} c) \theta$. So by $(\phi),\left(L(w) f\left(f I_{1}(w) f\right)^{-1} f W_{1}(w)\right) \theta=(b f f c) \theta=w \theta$. To get the second equality pre-multiply by $f$ throughout the proof.

With the additional restrictions $b$ and $I_{2}(w)$ exist. To prove the result for $\left(f W_{1}(w)\right) \theta$ proceed as above, using $(\theta 3)$ if $I_{2}(w)$ is an indicator of $b$ or $(\theta 5)$ applied to $M_{1}(w)$ otherwise.

We now deduce a result for $\theta$ like Lemma 2.5(i).

Lemma 3.11. Let $w=x_{i} a \in F_{X}^{u}$ where $C(w)=Y, L(w)=x_{i} b$ and $a$ has initial segment $p$ such that $x_{i} p \neq \hat{f}$ and $\mathbf{p} \rho x_{i+1} \ldots x_{n+1}$ for some $i$. Then

(i) $w \theta=\left(L(w) f\left(f \mathbf{p}^{-1} b f\right)^{-1} f \mathbf{p}^{-1} a\right) \theta$ and $(f w) \theta=\left(f L(w) f\left(f \mathbf{p}^{-1} b f\right)^{-1} f \mathbf{p}^{-1} a\right) \theta$ and

(ii) if $r=x_{i} \ldots x_{n+1}$ and $w=r s$ then $\left(f r^{-1} w\right) \theta=(f s) \theta$.

Proof. (i) We may assume $w=w \theta_{2}$, since $w \theta_{2} \theta=w \theta, L\left(w \theta_{2}\right)=L(w)$ and $\left(f \mathbf{p}^{-1}\left(a \theta_{2}\right)\right) \theta=\left(f \mathbf{p}^{-1} a\right) \theta$. By Lemma 3.10(i) $\quad w \theta=\left(L(w) f\left(f I_{1}(w) f\right)^{-1} f W_{1}(w)\right) \theta$. If $W_{1}(w) \neq w$ then $L(w) \neq I_{1}(w)$ so $C(b)=Y$ and $L\left(\mathbf{p}^{-1} a\right)=\mathbf{p}^{-1} b$. Hence by Lemma $3.10(\mathrm{i})$ $\left(f \mathbf{p}^{-1} a\right) \theta=\left(f \mathbf{p}^{-1} b f\left(f I_{1}(w) f\right)^{-1} f W_{1}(w)\right) \theta$ and the result follows, using $(\phi)$. Suppose $W_{1}(w)=w$, so $L(w)=I_{1}(w)$. If $w=R(w)$ then $w$ is $\hat{f}$-excluded so by $(\theta 3)$ and $(\theta 4)$ (acting in particular on $R(w)) w \theta_{4}=L(w) f\left(f I_{1}(w) f\right)^{-1} f L(w) f\left(f \mathbf{p}^{-1} b f\right)^{-1} f \mathbf{p}^{-1} a$ and the result follows. If $w \neq R(w)$ then by Lemma 3.10

$$
w \theta=\left(L(w) f\left(f I_{1}(w) f\right)^{-1} f M_{1}(w) f\left(f I_{2}(w) f\right)^{-1} f W_{2}(w)\right) \theta .
$$

Let $M_{1}(w)=x_{i} c$. Then by $(\theta 4), \quad\left(f M_{1}(w) f\right) \theta=\left(f I_{1}(w) f\left(f \mathbf{p}^{-1} b f\right)^{-1} f \mathbf{p}^{-1} c f\right) \theta$. Since 
$L\left(\mathbf{p}^{-1} a\right)=\mathbf{p}^{-1} c$ then by Lemma 3.10(i) $\left(f \mathbf{p}^{-1} a\right) \theta=\left(f \mathbf{p}^{-1} c f\left(f I_{2}(w) f\right)^{-1} f W_{2}(w)\right) \theta$. Combining these expressions we get the result.

(ii) Let $L(w)=r t$. We first prove that $\left(f r^{-1} r f f\right) \theta=(f t f) \theta$. Since $L\left(r^{-1} r t\right)=r^{-1} r t$ then by $(\theta 3),(\phi)$ and $(\theta 7)$, we need to show $\left(r^{-1} r t\right)_{M M}=t_{M M}$. We have $t=d x$ for some $d$ and $x \in Y \backslash C(d)$ so $\left(r^{-1} r d x\right)_{M}=\left(r^{-1} r d\right)_{R} x=d_{R} x$ by Definition 3.5 and the dual of Lemma 3.4(ii). If $C(d x) \subset Y$ then by Lemma 3.4(iii) $(d x)_{M}=\left(d_{R} x\right)_{M}$. If $C(d x)=Y$ and $R(d x) \neq d x$ then by Definition $3.5(d x)_{M}=d_{R} x$. Suppose $C(d x)=Y$ and $R(d x)=d x$. We have $x_{i(d)} \ldots x_{n+1} d_{R} \rho x_{i(d)} \ldots x_{n+1} d$. If $C\left(d_{R}\right) \subset C(d)$ then by comparing right indicators we get $x_{k} \ldots x_{n} d_{R} \rho d$ for some $k \leq n$. But then $x_{i(d)} \ldots x_{n+1} d_{R} \rho x_{i(d)} \ldots x_{k-1}\left(x_{k} \ldots x_{n}\right)^{2} d_{R}$. This is not possible since there exists a homomorphism from $F_{X}^{u} / \rho$ onto the free cyclic group $G_{\{x\}}$ taking generators to $x$. Hence $C\left(d_{R}\right)=C(d)$ and $R\left(d_{R}\right) \rho R(d)=d$ so by its definition $d_{R}=R\left(d_{R}\right)$. Thus $d_{R} x$ and $d x$ are $\rho$-related $Y$-indicators and by Theorem 1.3(iii) and Definition $3.5\left(d_{R} x\right)_{M}=(d x)_{M}$. Thus in all cases $\left(r^{-1} r\right)_{M M}=t_{M M}$.

We have $\left(f r^{-1} w\right) \theta=(f s) \theta$ when $i=1$ or $n$, by $(\phi)$ and $(\theta 1)$. Assume the result for $i>j>1$. Let $i=j$ and proceed by induction. Since $r=x_{i} p$ then by comparing expressions for $w \theta$ from (i) and Lemma 3.10(i) we get $\left(\left(f I_{1}(w) f\right)^{-1} f W_{1}(w)\right) \theta=\left(\left(f p^{-1} b f\right)^{-1} f p^{-1} a\right) \theta=$ $\left((f t f)^{-1} f s\right) \theta$, by Lemma 3.7 and induction. So by Lemma 3.10(i) and the first part of the proof

$$
\left(f r^{-1} w\right) \theta=\left(f r^{-1} r f f\left(f I_{1}(w) f\right)^{-1} f W_{1}(w)\right) \theta=\left(f f f(f f f)^{-1} f s\right) \theta=(f s) \theta .
$$

Recall Assumption 3.3. We can now see that it is valid when $C(u)=Y$. Say $u=\left(x_{i} \ldots x_{n+1}\right)^{0} v$ where $C(u) \supset C(v)$. By Lemmas 3.10 and $3.11 u \theta=\left(L(u) f(f t f)^{-1} f v\right) \theta$ where $L(u)=\left(x_{i} \ldots x_{n+1}\right)^{0} t$. Since $C(v) \subset Y$ then $(f v) \theta=f v_{R}$, so $u \theta$ has final segment $v_{R}$.

Lemma 3.12. Suppose $a, b \in F_{X}^{u}, x, y \in Y \backslash C(a)$ and $a \rho b$. If xay is $a Y$-indicator or $Y$-link then $(f x a y f) \theta=(f x b y f) \theta$. If ay is a left $Y$-indicator then $($ ayf $) \theta=($ byf $) \theta$.

Proof. If $(\theta 4),(\theta 5)$ and $(\theta 6)$ do not vary the segments $x a y$ and $x b y$ then the result is easy to check, using Definition 3.5. A similar statement applies for left $Y$-indicators. Let $x a y$ be a $Y$-indicator. Since $(\theta 4)$ and $(\theta 5)$ do not vary $Y$-indicators assume $x=x_{i}, y=x_{i}$ and $a=p c q$ where $\mathbf{p} \rho x_{i+1} \ldots x_{n+1}$ and $\mathbf{q} \rho x_{0} \ldots x_{j-1}$ for some $i$ and $j$. Since $x a y$ is a $Y$-indicator then $i>j$ and $x a y$ is $\hat{f}$-excluded so by $(\theta 6),(\theta 7)$ and $(\phi),(f x a y f) \theta=$ $f\left(x a \mathbf{q}^{-1}\right)_{M} f\left(f\left(\mathbf{p}^{-1} a \mathbf{q}^{-1}\right)_{M} f\right)^{-1} f\left(\mathbf{p}^{-1} a y\right)_{M} f$. But by Corollary $1.4 b=r d s$ for some $r, d, s$ such that $\mathbf{r} \rho \mathbf{p}$ and $\mathbf{s} \rho \mathbf{q}$ (with $r=1$ or $s=1$ if and only if $p=1$ or $q=1$ respectively). By Lemma 3.4(iii) then $\left(x a \mathbf{q}^{-1}\right)_{M}=\left(x b \mathbf{s}^{-1}\right)_{M}, \quad\left(\mathbf{p}^{-1} a \mathbf{q}^{-1}\right)_{M}=\left(\mathbf{r}^{-1} b \mathbf{s}^{-1}\right)_{M}$ and $\quad\left(\mathbf{p}^{-1} a y\right)_{M}=$ $\left(\mathbf{r}^{-1} \text { by }\right)_{M}$ so $(f x a y f) \theta=(f x b y f) \theta$.

Now let xay be a $Y$-link with $x=y=x_{i}$ for some $i$, where $p$ is an initial segment of $a$, $\mathbf{p} \rho x_{i+1} \ldots x_{n+1}$ and $L(x a y)=x d$. By Lemma 3.11(i) $(f x a y f) \theta=\left(f x d f\left(f \mathbf{p}^{-1} d f\right)^{-1} f \mathbf{p}^{-1} a y f\right) \theta$. This equation still holds if $x p=\hat{f}$, by Lemma 3.11(ii) and $(\phi)$. We have $x b y=x q e$ and $L(x b y)=x g$ where $\mathbf{p} \rho \mathbf{q}$. Since $x d$ and $x g$ are $\rho$-related $Y$-indicators (so $d \rho g$ ) and $C\left(\mathbf{p}^{-1} d\right) \subset Y$ we need only show that $\left(f \mathbf{p}^{-1}\right.$ ayf $) \theta=\left(f \mathbf{q}^{-1}\right.$ byf $\left.f\right) \theta$. This follows since $\left(\mathbf{p}^{-1} \text { ay }\right)_{M}=$ $\left(\mathbf{p}^{-1} a\right)_{R} y=\left(\mathbf{q}^{-1} b\right)_{R} y=\left(\mathbf{q}^{-1} b y\right)_{M}$ by the dual of Lemma 3.4(ii). By an analysis similar to the first paragraph we get the left $Y$-indicator result. 
Lemma 3.13. Let $w \in F_{X}^{u}$ and $x \in Y$. Then

(i) $(x w) \theta=(x(w \theta)) \theta$ and (ii) $(f w) \theta=(f(w \theta)) \theta$.

Proof. (i) We may assume $w=w \theta_{2}$. Suppose $C(x w)=Y$ : otherwise the result follows by Assumption 3.1. By Theorem 1.3(iii) and Lemma $3.12\left(f L(x w) f\left(f I_{1}(x w) f\right)^{-1}\right) \theta=$ $\left(f L(x(w \theta)) f\left(f I_{1}(x(w \theta)) f\right)^{-1}\right) \theta$. So by Lemma 3.10 we need to show $\left(f W_{1}(x w)\right) \theta=$ $\left(f W_{1}(x(w \theta))\right) \theta$. If $W_{1}(x w) \neq x w$ then $I_{1}(x w)=I_{1}(w)$. Using Theorem 1.3(iii) it is easy to check, since $w \theta \rho w$ that $I_{1}(x(w \theta))=I_{1}(w \theta)$. Then $W_{1}(x w)=W_{1}(w)$ and $W_{1}(x(w \theta))=$ $W_{1}(w \theta)$. Equating the expressions for $w \theta$ and $w \theta \theta$ from Lemma 3.10(i) we get $\left(f W_{1}(w)\right) \theta=\left(f W_{1}(w \theta)\right) \theta$ and hence the result. If $W_{1}(x w)=x w=R(x w)$ we get the result by the dual of Lemma 3.12 .

Suppose $W_{1}(x w)=x w \neq R(x w)$. If $\hat{f}$ is not an initial segment of $x w$ then by Lemma $3.10(\mathrm{ii}),\left(f W_{1}(x w)\right) \theta=\left(f M_{1}(x w) f\left(f I_{2}(x w) f\right)^{-1} f W_{2}(x w)\right) \theta$. This equation also holds if $x w=$ $\hat{f} a$. To see this let $M_{1}(x w)=\hat{f} b$. If $W_{2}(x w)=x_{i} \ldots x_{n} a$ and $I_{2}(x w)=x_{i} \ldots x_{n} b$ for some $i \leq n$ then $\left(f W_{2}(x w)\right) \theta=\left(f I_{2}(x w) f(f b f)^{-1} f a\right) \theta$ by Lemma 3.11. Alternatively if $W_{2}(x w)$ is a segment of $a$, by Lemma 3.10(i) $(f a) \theta=\left(f b f\left(f I_{2}(x w) f\right)^{-1} f W_{2}(x w)\right) \theta$, and since $\left(f W_{1}(x w)\right) \theta=(\hat{f} f a) \theta$ we get the equation. Observe that since $W_{1}(x w)=x w$ then $I_{2}(x w)=$ $I_{1}(w)$ so by Lemma 3.10(i), $w \theta=\left(L(w) f\left(f I_{1}(w) f\right)^{-1} f W_{2}(x w)\right) \theta$. Likewise $w \theta \theta=$ $\left.L(w \theta) f\left(f I_{1}(w \theta) f\right)^{-1} f W_{2}(x(w \theta))\right) \theta$. Since $w \theta=w \theta \theta$ then by Lemma $3.12\left(f W_{2}(x w)\right) \theta=$ $\left(f W_{2}(x(w \theta))\right) \theta$. Hence since $M_{1}(x w)=x L(w) \rho x L(w \theta)=M_{1}(x(w \theta))$, then by Lemma 3.12, $\left(f W_{1}(x w)\right) \theta=\left(f W_{1}(x(w \theta))\right) \theta$.

(ii) It follows by straightforward induction, based on (i), that $(\hat{f} w) \theta=\left(x_{1} \ldots x_{n} w\right) \theta=$ $\left(x_{1} \ldots x_{n}(w \theta)\right) \theta=(\hat{f}(w \theta)) \theta$. It is easily seen that $\left(\hat{f}^{-1}\right) \theta=\hat{f}^{-1}$. So by Lemma 3.7

$$
(f(w \theta)) \theta=\left(\hat{f}^{-1} \hat{f}(w \theta)\right) \theta=\left(\hat{f}^{-1}(\hat{f}(w \theta)) \theta\right) \phi=\left(\hat{f}^{-1}(\hat{f} w) \theta\right) \phi=\left(\hat{f}^{-1} \hat{f} w\right) \theta=(f w) \theta .
$$

The next result is the key lemma of the paper. It will be used to show that $\left\{w \theta ; w \in F_{X}^{u}\right\}$ with multiplication $u \theta \cdot v \theta=(u \theta(v \theta)) \theta$ is a semigroup.

Lemma 3.14. Let $u, v \in F_{X}^{u}$ where $C(u v) \subseteq Y$. Then $(u v) \theta=(u \theta(v \theta)) \theta$.

Proof. The result is immediate if $C(u v) \subset Y$ (by Assumption 3.1), or if $|C(u)|=1$ (by Lemma 3.13(i)) since then $u \theta=u$ by Assumption 3.1. Assume the result for $C(u) \subset \mathbf{U}$, some $\mathbf{U} \subseteq Y$, and proceed by induction. Suppose $C(u)=\mathbf{U}$ and $C(u v)=Y$.

Suppose $C(u) \subset Y$. Then $L(u v)=u v_{1}$ and $L(u \theta(v \theta))=u \theta v_{2}$ where $v_{1} \rho v_{2}$ by Corollary 1.4. Either $I_{1}(u v)=R L(u v)=u_{1} v_{1}$ and $I_{1}(u \theta(v \theta))=u_{2} v_{2}$ where $u_{1} \rho u_{2}$ by Corollary 1.4 or $I_{1}(u v)=I_{1}(v) \rho I_{1}(v \theta)=I_{1}(u \theta(v \theta)$ ) (see Theorem 1.3(iii)). By Lemmas 3.10(i) and 3.12 we need to show $\left(f W_{1}(u v)\right) \theta=\left(f W_{1}(u \theta(v \theta))\right) \theta$. If $I_{1}(u v)=u_{1} v_{1}$ then $W_{1}(u v)=u_{1} v$ and $W_{1}(u \theta(v \theta))=u_{2}(v \theta)$; also $u_{1}=x a_{1}, u_{2}=x a_{2}$ for some $x \in Y \backslash C\left(a_{1}\right)$ and $a_{1} \theta=a_{2} \theta$ by Assumption 3.1 and Theorem 1.3(iii). By Lemmas 3.13, 3.8 and the induction assumption $\left(f W_{1}(u v)\right) \theta=\left(f x a_{1} v\right) \theta=\left(f\left(x a_{1} v\right) \theta\right) \theta=\left(f\left(x\left(a_{1} v\right) \theta\right) \theta\right) \theta=\left(f\left(x\left(a_{1} \theta(v \theta)\right) \theta\right) \theta\right) \theta=$ $\left(f\left(x\left(a_{2} \theta(v \theta)\right) \theta\right) \theta\right) \theta=\left(f\left(x\left(a_{2}(v \theta)\right) \theta\right) \theta\right) \theta=\left(f\left(x a_{2}(v \theta)\right) \theta\right) \theta=\left(f x a_{2}(v \theta)\right) \theta=\left(f W_{1}(u \theta(v \theta))\right) \theta$. Alternatively $I_{1}(u v)=I_{1}(v)$, so $W_{1}(u v)=W_{1}(v)$. Since $L(v) \rho L(v \theta)$ and $I_{1}(v) \rho I_{1}(v \theta)$, 
then equating the expressions from Lemma 3.10(i) for $v \theta$ and $v \theta \theta$, using Lemma 3.12, we get $\left(f W_{1}(u v)\right) \theta=\left(f W_{1}(u \theta(v \theta))\right) \theta$.

Now suppose $C(u)=Y$ and $u \theta_{3}=a \hat{f} w$ where $w$ is an $\hat{f}$-excluded segment or $w=1$. Clearly $(u v) \theta=\left(u \theta_{3}\left(v \theta_{3}\right)\right) \theta$, so by Lemmas 3.7 and 3.9 we need only prove $(\hat{f} w v) \theta=$ $((f w) \theta(v \theta)) \theta$, or equivalently $(f w v) \theta=((f w) \theta(v \theta)) \theta$. If $C(w) \subset Y$ then, with $p=$ $x_{i(w)} \ldots x_{n+1}$, we get $(f w v) \theta=\left(f p^{-1} p w v\right) \theta=\left(f\left(p^{-1} p w v\right) \theta\right) \theta=\left(f\left(\left(p^{-1} p w\right) \theta(v \theta)\right) \theta\right) \theta=$ $\left(f\left(\left(p^{-1} p w_{R}\right) \theta(v \theta)\right) \theta\right) \theta=\left(f\left(p^{-1} p w_{R}(v \theta)\right) \theta\right) \theta=\left(f p^{-1} p w_{R}(v \theta)\right) \theta=\left(f w_{R}(v \theta)\right) \theta=((f w) \theta(v \theta)) \theta$ by Lemmas 3.11(ii), 3.13(ii), 3.8, the induction assumption, Definition 3.2 and $(\theta 7)$. Now suppose $C(w)=Y$, so $w=R(w)=x b$ for some $x \in Y \backslash C(b)$. If $(\theta 4),(\theta 5)$ and $(\theta 6)$ do not vary $w$ then $(f w v) \theta=(f(x b v) \theta) \theta=(f(x((b \theta)(v \theta)) \theta) \theta) \theta=(f(x(b \theta)(v \theta)) \theta) \theta=(f x(b \theta)$ $(v \theta)) \theta=((f w) \theta(v \theta)) \theta$ by Lemma 3.13, the induction assumption, Definition 3.5 and $(\theta 7)$. Finally suppose $w=x_{i} b, L(w)=x_{i} c$, and $p$ is an initial segment of $b$ such that $\mathbf{p} \rho x_{i+1} \ldots x_{n+1}$ for some $i$. By Lemma $3.11(f w v) \theta=\left(f x_{i} c f\left(f \mathbf{p}^{-1} c f\right)^{-1} f \mathbf{p}^{-1} b v\right) \theta$. Since $C\left(\mathbf{p}^{-1} b\right) \subset Y$ then by the above $\left(f \mathbf{p}^{-1} b v\right) \theta=\left(\left(f \mathbf{p}^{-1} b\right) \theta(v \theta)\right) \theta$. But by Lemma 3.11(i), $(f w) \theta=\left(f x_{i} c f\left(f \mathbf{p}^{-1} c f\right)^{-1} f \mathbf{p}^{-1} b\right) \theta$ so $(f w v) \theta=((f w) \theta(v \theta)) \theta$.

REMARK. We have not yet shown, for $C(w)=Y$, that $w \theta$ is a unique representative of the class $w \rho$. This will follow from Theorem 4.1.

\section{A model for $F_{X}^{c r}$.}

THEOREM 4.1. Let $S=\left\{w \theta ; w \in F_{X}^{u}\right\}$ with a binary operation defined by $u \theta \cdot v \theta=$ $(u \theta(v \theta)) \theta$. Then $S \cong F_{X}^{c r}$.

Proof. For any $u, v, w \in F_{X}^{u}$ we have by Lemma 3.14 that $u \theta \cdot v \theta=(u v) \theta$ so $(u \theta \cdot v \theta) \cdot w \theta=(u v) \theta \cdot w \theta=(u v w) \theta=u \theta \cdot(v w) \theta=u \theta \cdot(v \theta \cdot w \theta)$. Hence $S$ is a semigroup.

We will now check that $S$ is completely regular. For any $u, v \in F_{X}^{u}$, since $u \theta \rho u$ then by Theorem 1.3(iii) $u \theta \mathscr{L} v \theta$ only if $L(u) \rho L(v)$. Conversely suppose $L(u) \rho L(v)$. Since $L(u \theta) \rho L(u)$, assume $u=u \theta$ and $v=v \theta$. By Lemma 3.10(i) $u \theta=$ $\left(L(u) f\left(f I_{1}(u) f\right)^{-1} f W_{1}(u)\right) \theta$, by Lemma $3.12 \quad(L(u) f) \theta=(L(v) f) \theta$ and by $(\theta 2)$ and Lemma $3.9\left(f W_{1}(u)\left(f W_{1}(u) f\right)^{-1}\right) \theta=\left(f W_{1}(u) f\left(f W_{1}(u) f\right)^{-1} f\right) \theta=f$. So with $a=$ $\left(f W_{1}(u) f\right)^{-1} f I_{1}(u) f\left(f I_{1}(v) f\right)^{-1} f W_{1}(v)$ we get by $(\phi)$ and Lemma $3.10(i)$ that $u \theta \cdot a \theta=$ (ua) $\theta=v \theta$. Hence $u \theta \mathscr{L} v \theta$ if and only if $L(u) \rho L(v)$. There is a dual result for $\mathscr{R}$. But then $u \theta \mathscr{H}\left(L(u) f(f R(u) L(u) f)^{-1} f R(u)\right) \theta$, which is an idempotent. So $S$ is a union of groups. We have $u^{-1} \theta=(u \theta)^{-1}$ in $S$ by Theorem 1.3(iii) and $(\theta 2)$.

By Lemma $3.14 S$ is generated by $\{x \theta ; x \in X\}$. So by the free property of $F_{X}^{u} / \rho \cong F_{X}^{\text {cr }}$ there is a surjective homomorphism $\alpha: F_{X}^{u} / \rho \rightarrow S$ given by $(x \rho) \alpha=x \theta$ for all $x \in X$. By the definition of multiplication in $S$ and Lemma 3.14 then $(w \rho) \alpha=w \theta$ for all $w \in F_{X}^{u}$. Since $w \theta \rho w$ then $\alpha$ is injective, so $\alpha$ is an isomorphism.

Notice that since $\alpha$ in this proof is an isomorphism then, for each $w \in F_{X}^{u}, w \theta$ is a unique representative of $w \rho$. This is in accordance with Assumption 3.1.

Some properties of the model $S$ for $F_{X}^{c r}$ can be easily deduced. Recall the definitions of section 1 and Definitions 2.6, 3.2 and 3.5. We first characterize the $\hat{f}$-bounded segments of an element of $S$ of content $Y$. 
Define $a \in F_{X}^{u}$ to be $Y$-basic if and only if $a$ satisfies the following properties.

(i) $C(a) \subseteq Y, a=a_{M M}$ and $a$ is $\hat{f}$-excluded.

(ii) Suppose $p, q \in F_{X}^{u}$ where $\left(\mathbf{p}, x_{i} \ldots x_{n}\right) \in \rho$ and $\left(\mathbf{q}, x_{1} \ldots x_{j}\right) \in \rho$ for some $i \geq 1$ and $j \leq n$. If $C(a)=Y$ and $a=p b$ or $a=b q$ for some $b$ then $a$ is a left or right $Y$-indicator respectively. If $C(a)=Y$ then $a \neq p d q$ for any $d$.

Define $a \in F_{X}^{u 1}$ to be left (right) $Y$-basic if and only if $a$ satisfies (ii) and

(i') $C(a) \subseteq Y, a=a_{L}$ (respectively $a_{R}$ ), and $a$ is $\hat{f}$-excluded.

Define $H_{Y}$ to be the subgroup of $G_{Y}$ freely generated by $\{f a f, \hat{f} ; a$ is $Y$-basic $\}$.

Define $D_{Y}=\left\{u f h f v ; u\right.$ and $v$ are respectively left $Y$ - and right $Y$-basic and $\left.h \in H_{Y}\right\}$.

Corollary 4.2. Let $w \in F_{X}^{u}$ and $C(w)=Y$. Then there is a unique left $Y$-basic $u, a$ unique right $Y$-basic $v$ and a unique $h \in H_{Y}$ such that $w \theta=u$ fhfv. In $S$ the $\mathscr{D}$-class of $w \theta$ is $\{r \theta ; C(r)=C(w)\}=D_{Y}$, the $\mathscr{R}$-class of $w \theta$ is $\{r \theta ; L(r \theta)=L(w \theta)\}$, the $\mathscr{L}$-class of $w \theta$ is $\{r \theta ; R(r \theta)=R(w \theta)\}$, and the $\mathscr{H}$-class of $w \theta$ is the free group $u f H_{Y} f v$.

Proof. The expression for $w \theta$ follows from its definition; $u$ is the $\hat{f}$-bounded initial segment of $w \theta$ if it exists, otherwise $u=1$ (there is a dual statement for $v$ ). By Theorem 1.3(ii), $\{r \theta ; C(r)=C(w)\}$ is the $\mathscr{D}$-class of $w \theta$. It can be directly checked that the free generators of $H_{Y}$ are in $S$ (by a proof along the lines of that for Lemma 3.8), as are $u f$ and fv for any left $Y$-basic $u$ and right $Y$-basic $v$. So by Lemma 3.7, $D_{Y} \subset S$ and by Theorem 1.3(ii), $D_{Y}$ is the $\mathscr{D}$-class of $w \theta$. The $\mathscr{L}$ and $\mathscr{R}$-class characterizations are by Lemmas 3.10(i) and 3.12. The $\mathscr{H}$-class characterization then follows by the definition of $D_{Y}$.

Notice that by Theorem 4.1 , the construction of $w \theta$ from $w \in F_{X}^{u}$ may be simplified by replacing $w$ by an alternative $\rho$-related element.

We observe that the representative $w \theta$ of the $\rho$-class of $w \in F_{X}^{u}$ is uniquely defined modulo the choice of $u \theta$ for all $u \in F_{X}^{u}$ where $C(u) \subset C(w)$. To see this first note that the operations $(\theta 1), \ldots,(\theta 6)$ and $(\phi)$ just manipulate the spelling of $w$. By Definitions 3.2 and 3.5 the application of $(\theta 7)$ requires knowledge of the spelling of $u \theta$ for some $u \in F_{X}^{u}$, $C(u) \subset C(w)$.

As mentioned in the introduction our characterization of $F_{X}^{c r}$ is different from that of Gerhard [4]. He determines a set of free generators of $\left(H_{Y}\right) \rho$ that are unique up to solution of the word problem in $F_{X}^{u} / \rho$ for words of content less than $Y$. By this approach. he gets many expressions of the form $(f a f) \rho, f a f \in F_{X}^{u}$, for a generator. It is difficult to determine, using the solution to the word problem for words of content less than $Y$, whether two of these expressions denote the same generator. Gerhard's model for $F_{X}^{\mathrm{cr}}$, based on Petrich's structure theorem for completely regular semigroups [6, Theorem 3], is a union of Rees matrix semigroups. The Rees matrix semigroup corresponding to the $\mathscr{D}$-class of elements of content $Y$ has structure group $\left(H_{Y}\right) \rho$.

\section{REFERENCES}

1. A. H. Clifford, Semigroups admitting relative inverses, Ann. of Math. (2) 42 (1941), 1037-1049. 
2. A. H. Clifford, The free completely regular semigroup on a set, J. Algebra 59 (1979), $434-451$.

3. J. A. Gerhard, Free completely regular semigroups I: Representation, J. Algebra 82 (1983), 135-142.

4. J. A. Gerhard, Free completely regular semigroups II: Word problem, J. Algebra 82 (1983), 143-156.

5. D. B. McAlister, A homomorphism theorem for semigroups, J. London Math. Soc. $\mathbf{4 3}$ (1968), 355-366.

6. M. Petrich, The structure of completely regular semigroups, Trans. Amer. Math. Soc. 189 (1974), 211-236.

7. M. Petrich, Certain varieties and quasivarieties of completely regular semigroups, Canad. $J$. Math. 29 (1977), 1171-1197.

UNIVERSTTY OF TASMANIA

HOBART

TASMANIA

Australia 